\title{
Timelike boundary Liouville theory
}

\section{Citation}

Gutperle, Michael, and Andrew Strominger. 2003. "Timelike Boundary Liouville Theory." Physical Review D 67 (12). https://doi.org/10.1103/physrevd.67.126002.

\section{Permanent link}

http://nrs.harvard.edu/urn-3:HUL.InstRepos:41417257

\section{Terms of Use}

This article was downloaded from Harvard University's DASH repository, and is made available under the terms and conditions applicable to Other Posted Material, as set forth at http:// nrs.harvard.edu/urn-3:HUL.InstRepos:dash.current.terms-of-use\#LAA

\section{Share Your Story}

The Harvard community has made this article openly available.

Please share how this access benefits you. Submit a story.

Accessibility 
hep-th/0301038

SU-ITP-02/50

\title{
Timelike Boundary Liouville Theory
}

\author{
Michael Gutperle* and Andrew Strominger ${ }^{\dagger}$
}

\begin{abstract}
The timelike boundary Liouville (TBL) conformal field theory consisting of a negative norm boson with an exponential boundary interaction is considered. TBL and its close cousin, a positive norm boson with a non-hermitian boundary interaction, arise in the description of the $c=1$ accumulation point of $c<1$ minimal models, as the worldsheet description of open string tachyon condensation in string theory and in scaling limits of superconductors with line defects. Bulk correlators are shown to be exactly soluble. In contrast, due to OPE singularities near the boundary interaction, the computation of boundary correlators is a challenging problem which we address but do not fully solve. Analytic continuation from the known correlators of spatial boundary Liouville to TBL encounters an infinite accumulation of poles and zeros. A particular contour prescription is proposed which cancels the poles against the zeros in the boundary correlator $d(\omega)$ of two operators of weight $\omega^{2}$ and yields a finite result. A general relation is proposed between two-point CFT correlators and stringy Bogolubov coefficients, according to which the magnitude of $d(\omega)$ determines the rate of open string pair creation during tachyon condensation. The rate so obtained agrees at large $\omega$ with a minisuperspace analysis of previous work. It is suggested that the mathematical ambiguity arising in the prescription for analytic continuation of the correlators corresponds to the physical ambiguity in the choice of open string modes and vacua in a time dependent background.
\end{abstract}

\footnotetext{
* Dept. of Physics, Stanford University, Stanford CA. On leave of absence from Dept. of
} Physics and Astronomy, UCLA, Los Angeles, CA.

$\dagger$ Jefferson Physical Laboratory, Harvard University, Cambridge MA 


\section{Contents}

1. Introduction .. . . . . . . . . . . . . . . . . . . . . . . . . 1

2. TBL and String Theory . . . . . . . . . . . . . . . . . . . . . . . 5

2.1. Minisuperspace Review . . . . . . . . . . . . . . . . . . . . . . . . . . . 5

2.2. Two-Point Function as Stringy Bogolubov Coefficient . . . . . . . . . . . . . . 7

3. Bulk Correlators . . . . . . . . . . . . . . . . . . . . . . . . . . . 10

3.1. One-Point Function . . . . . . . . . . . . . . . . . . . . . . . . . . . . . 12

3.2. Boundary States . . . . . . . . . . . . . . . . . . . . . . . . . . . . . . 13

3.3. N-Point Correlators . . . . . . . . . . . . . . . . . . . . . . . . . . . . . 15

4. Boundary Correlators . . . . . . . . . . . . . . . . . . . . . . . . . . . . . 16

4.1. Spacelike Boundary Liouville . . . . . . . . . . . . . . . . . . . . . . . . 16

4.2. Analytic Continuation to Timelike Boundary Liouville . . . . . . . . . . . . . 17

Appendix A. Properties of special functions . . . . . . . . . . . . . . . . . . . . . 23

\section{Introduction}

In this paper we study the two-dimensional conformal field theory described by a $c=1$ negative norm boson with an exponential interaction on the boundary. The action is

$$
S_{T B L}=-\frac{1}{2 \pi} \int_{\Sigma} \partial X \bar{\partial} X+\frac{\lambda}{2} \int_{\partial \Sigma} e^{X}
$$

We will refer to this as the TBL (timelike boundary Liouville) theory. Due to the 'wrong' sign in front of the kinetic term in (1.1), the $X$ correlator on the upper half plane is 1

$$
\langle X(z, \bar{z}) X(w, \bar{w})\rangle=\ln |z-w|+\ln |z-\bar{w}|
$$

and the boundary interaction is marginal. This wrong sign also implies that the functional integral

$$
\int \mathcal{D} X e^{-S_{T B L}}
$$

is not well-defined. In order to define the theory, we will need to specify some kind of analytic continuation procedure.

1 Here and in the rest of the paper we have set $\alpha^{\prime}=1$. 
TBL has not been previously studied in much detail. several theories which have been well-studied. Analytically continuing $X \rightarrow i \phi$ we obtain a free positive-norm boson with a non-hermitian boundary interaction [3, 4]

$$
S_{N H}=\frac{1}{2 \pi} \int_{\Sigma} \partial \phi \bar{\partial} \phi+\frac{\lambda}{2} \int_{\partial \Sigma} e^{i \phi} .
$$

This can be viewed as 'half' the boundary Sine-Gordon theory (with a marginal boundary interaction) [5, 6, [7], which has a $\lambda e^{i \phi}+\bar{\lambda} e^{-i \phi}$ boundary interaction. The correlators of TBL are hence formally related to those following from (1.4). However since (1.4) has a non-hermitian interaction, its correlators are also not unambiguously defined.

Generalizing the coefficient of the exponent in (1.4), and improving the stress tensor so that the interaction remains marginal, we obtain the ordinary spacelike boundary Liouville (SBL) theory

$$
S_{S B L}=\frac{1}{2 \pi} \int_{\Sigma} \partial \phi \bar{\partial} \phi+\frac{\lambda}{2} \int_{\partial \Sigma} e^{b \phi} .
$$

This theory has been studied for generic real values of $b$. The two-point boundary correlators have been explicitly obtained in [8,9], the bulk-boundary correlators are in [10], an integral form of the three-point boundary correlators has been given in [1], and supersymmetric correlators are in [12]. We shall see that the analytic continuation $b \rightarrow i$ from $S_{S B L}(1.5)$ to $S_{N H}(1.4)$ and $S_{T B L}(1.1)$ is highly non-trivial and encounters ambiguities for boundary correlators. This difficulty has been encountered previously while studying the role of $S_{N H}$ in the $c=1$ accumulation point of minimal models 4 , 13. In this paper we will give a specific, physically-motivated (in the context of string theory) proposal for continuing the two-point boundary correlator to TBL. The prescription involves approaching the TBL theory through $c<1$ theories with a linear dilaton.

Yet another related theory is the timelike boundary Sine-Gordon theory

$$
S_{T B S G}=-\frac{1}{2 \pi} \int_{\Sigma} \partial X \bar{\partial} X+\int_{\partial \Sigma}\left(\lambda_{-} e^{-X}+\lambda_{+} e^{X}\right) .
$$

2 A minisuperspace analysis appeared in 洎. Certain bulk one-point functions were recently computed in [2]. 
The boundary state for this theory was found by analytic continuation from the spacelike case in [14,15], in the context of string theory, where some cases describe an s-brane [16]. Aspects of closed string emission were computed in [17, 18, 19, 20]. We expect the boundary correlators for this theory, which have not been computed, to be more intricate due to the extra interaction term. A further complication is that there is no "free" region at $X \rightarrow-\infty$ in which the (open string) spectrum can be easily understood. However the extra interaction term could also simplify matters by controlling divergences and leading to a hermitian action for $X \rightarrow i \phi$. We will not consider this interesting theory further in the present paper. 目

The theories described by the actions $S_{T B L}$ and $S_{N H}$ are of interest in a number of contexts. In string theory $S_{T B L}$ is the worldsheet action describing time-dependent open string tachyon condensation [14,1],16]. This can equivalently be viewed as unstable D-brane decay or the future half of an s-brane. This relation will be further discussed in section 2. Non-hermitian boundary interactions of the general variety $S_{N H}$ (1.4) are realized in a variety of condensed matter systems [22]. $S_{N H}$ itself arises in the infrared limit of a $2 \mathrm{~d}$ superconductor with a magnetic field and a line defect which are not parallel [23]. The non-hermiticity corresponds to a lack of reflection symmetry across the defect. Finally (1.4) is related to the $c=1$ theory obtained as the accumulation point of the $c<1$ unitary boundary minimal models, all of which it in a sense contains [4. 13]. This highlights the non-trivial nature of this CFT.

As noted above, the TBL theory is not well-defined without some kind of additional prescription. This prescription should be dictated by, and may depend on, the physical context in which the theory arises. In the context of string theory, we shall argue that the two-point correlator gives stringy Bogolubov coefficients describing quantum open string creation during tachyon condensation. The creation rate depends only on the magnitude of the two-point function, which was computed in the minisuperspace approximation to TBL

3 A minisuperspace analysis will appear in 21]. 
in [1]. We find that a natural prescription for defining the TBL two-point correlators by analytic continuation gives a result in agreement with the minisuperspace approximation at high energies. This connection further suggests that the mathematical ambiguity in the correlator corresponds physically to the ambiguity in the choice of a vacuum state and modes for open strings during the time-dependent process of tachyon condensation.

We wish to stress that we regard this work as a preliminary step in defining the TBL CFT. We have not given a procedure for defining the boundary three-point function (known only in integral form for the spacelike case), or verified that our prescription yields correlators obeying the properties of a CFT. Indeed since the TBL theory is not unitary it is not clear what those properties should be. Further, we feel there is some hidden "meaning" in the (accumulation of) singularities which we have not fathomed. We regard all of these as interesting problems for future investigation. Since tachyon condensation is an allowed process in string theory we believe that, despite the apparently singular behavior of the TBL theory, a well-defined set of correlators should exist.

An intriguing feature of our continuation prescription to TBL is the following. As mentioned above, an intermediate step involves $c<1$ timelike linear dilaton theories, which are of interest in their own right. The proposed prescription determines the norm of the boundary correlator $|d(\omega)|$ for all real values of the dilaton. Interestingly, as detailed in section 4.2, the phase $\operatorname{Im}[\ln d(\omega)]$ is determined only for 'rational' values of the dilaton, and does not have a smooth extension to real values.

This paper is organized as follows. In section 2.1 the minisuperspace analysis of TBL in the context of string theory is reviewed. While the validity of the minisuperspace analysis is not a priori obvious, it gives us invaluable clues as to which operators to consider and what kind of phenomena to expect. In section 2.2 a general relation between CFT two-point functions and stringy Bogolubov coefficients is proposed. Section 3 describes the

4 It would be interesting to understand the physical origin/resolution of these ambiguities in the superconductor context [23], where to date largely bulk quantities have been considered. 
computation of correlators of bulk operators, and explicitly works out the one and twopoint functions as well as the boundary state. In section 4.1 we review the crucial results of [8] on the boundary two-point function for ordinary boundary Liouville theory which has a spacelike boson. Finally in 4.2 we detail our proposal for continuing this two-point function to TBL. This involves contours for analytic continuation of the background charge (i.e. a timelike linear dilaton) and $\omega$, as well as a prescription to sum a certain series of residues after the analytic continuation. The final result for the magnitude gives agreement at high energies with the minisuperspace computation of the open string creation rate. Properties of some special functions appearing in the expressions for correlators are given in an appendix.

\section{TBL and String Theory}

TBL is the worldsheet description of a time dependent process in which the open string tachyon field $\mathcal{T}=e^{X^{0}}$ starts at its unstable minimum in the infinite past $5 X^{0}=-\infty$ and then rolls to an infinite value in the far future $X^{0}=+\infty$. Such processes have been discussed in [16, 14, 1]. This may equivalently be described as the decay of an unstable brane or the future half of an s-brane (which consists of creation of an unstable brane followed by its decay).

\subsection{Minisuperspace Review}

The minisuperspace approximation to TBL was described in [1] and will be reviewed in this subsection. While the validity of this approximation is not a priori obvious, it nevertheless provides invaluable clues as to what to look for in the exact treatment.

The $L_{0}=0$ constraint on the open string worldsheet for a half s-brane becomes a

5 In classical string theory the tachyon can be perched indefinitely at its unstable minimum without being pushed off by quantum fluctuations. 
Schrödinger equation for the open string wave functions $\|^{6}$

$$
\left(\frac{\partial^{2}}{\partial X^{2}}+\lambda e^{X}+N-1+\vec{p}^{2}\right) \psi(X)=0
$$

Here we abbreviate the timelike coordinate $X^{0}$ as $X, \vec{p}$ is the spatial momentum and $N$ is the oscillator level number. The solutions to this are Bessel functions

$$
\psi_{\vec{p}}^{i n}=\frac{\lambda^{i \omega}}{\sqrt{2 \omega}} \Gamma(1-2 i \omega) e^{i \vec{p} \cdot \vec{x}} J_{-2 i \omega}\left(2 \sqrt{\lambda} e^{X / 2}\right), \quad \omega \equiv \sqrt{N-1+\vec{p}^{2}}
$$

and their complex conjugates. In the far past this solution approaches a positive frequency plane wave

$$
X \rightarrow-\infty, \quad \psi_{\vec{p}}^{i n} \rightarrow \frac{1}{\sqrt{2 \omega}} e^{-i \omega X+i \vec{p} \cdot \vec{x}}
$$

In the far future $X \rightarrow \infty$,

$$
\psi_{\vec{p}}^{i n} \rightarrow \frac{\lambda^{i \omega-1 / 4} \Gamma(1-2 i \omega)}{\sqrt{8 \pi \omega}} e^{-X / 4+i \vec{p} \cdot \vec{x}}\left(e^{\pi \omega-2 i \sqrt{\lambda} e^{X / 2}+i \frac{\pi}{4}}+e^{-\pi \omega+2 i \sqrt{\lambda} e^{X / 2}-i \frac{\pi}{4}}\right) .
$$

We see that the incoming modes $\psi_{\vec{p}}^{\text {in }}$ contain both negative and positive frequency parts in the far future. This indicates open string pair creation. Normalized outgoing positive frequency modes are Hankel functions

$$
\psi_{\vec{p}}^{\text {out }}=\sqrt{\frac{\pi}{2 i}} e^{-\pi \omega+i \vec{p} \cdot \vec{x}} H_{-2 i \omega}^{(2)}\left(2 \sqrt{\lambda} e^{X / 2}\right) \rightarrow \frac{\lambda^{-1 / 4}}{\sqrt{2}} e^{-\frac{X}{4}-2 i \sqrt{\lambda} e^{X / 2}+i \vec{p} \cdot \vec{x}}, \quad X \rightarrow \infty
$$

The in and out modes are related by the Bogolubov transformation

$$
\begin{aligned}
\psi_{\vec{p}}^{\text {out }} & =\alpha_{\vec{p}} \psi_{\vec{p}}^{\text {in }}+\beta_{\vec{p}} \psi_{-\vec{p}}^{\text {in* }}, \\
\psi_{\vec{p}}^{i n} & =\alpha_{\vec{p}}^{*} \psi_{\vec{p}}^{\text {out }}-\beta_{\vec{p}} \psi_{-\vec{p}}^{\text {out* }}, \\
\alpha_{\vec{p}} & =\frac{\lambda^{-i \omega}}{\sqrt{4 \pi i \omega}} \Gamma(1+2 i \omega) e^{\pi \omega}, \\
\beta_{\vec{p}} & =-\frac{\lambda^{i \omega}}{\sqrt{4 \pi i \omega}} \Gamma(1-2 i \omega) e^{-\pi \omega} .
\end{aligned}
$$

\footnotetext{
6 In our conventions $\alpha^{\prime}=1$.
} 
which obey $\alpha_{\vec{p}} \alpha_{\vec{p}}^{*}-\beta_{\vec{p}} \beta_{\vec{p}}^{*}=1$ as required. Expanding

$$
\phi=\sum_{\vec{p}}\left(\psi_{\vec{p}}^{i n} a_{\vec{p}}^{i n}+\psi_{\vec{p}}^{i n *} a_{\vec{p}}^{i n \dagger}\right)=\sum_{\vec{p}}\left(\psi_{\vec{p}}^{o u t} a_{\vec{p}}^{\text {out }}+\psi_{\vec{p}}^{\text {out } *} a_{\vec{p}}^{\text {out } \dagger}\right)
$$

the in vacuum becomes

$$
\mid \text { in }>=\prod_{\vec{p}}\left(1-\left|\gamma_{\omega}^{\text {in }}\right|^{2}\right)^{1 / 4} e^{-\frac{1}{2} \sum \gamma_{\omega}^{\text {in }}\left(a_{\vec{p}}^{\text {out } \dagger}\right)^{2}} \mid \text { out }>,
$$

where

$$
\gamma_{\omega}^{i n}=\frac{\beta_{\vec{p}}^{*}}{\alpha_{\vec{p}}}=-i e^{-2 \pi \omega},
$$

where $\omega$ and $\vec{p}$ are related by $(2.2)$. The in vacuum is annihilated by $a_{\vec{p}}^{\text {in }}=\alpha_{\vec{p}} a_{\vec{p}}^{\text {out }}+\beta_{\vec{p}}^{*} a_{-\vec{p}}^{\text {out }}$. Relation (2.8) expresses the fact that if there are no incoming particles at $X \rightarrow-\infty$, there will necessarily be outgoing particles at $X \rightarrow \infty$. $\alpha$ and $\beta$ can be changed by phase redefinitions of the modes, but the total string creation for a mode with frequency $\omega$ is characterized by $\left|\gamma_{\vec{p}}\right|$. Similarly the out vacuum is an excited state of the in vacuum

$$
\mid \text { out }>=\prod_{\vec{p}}\left(1-\left|\gamma_{\omega}^{\text {out }}\right|^{2}\right)^{1 / 4} e^{-\frac{1}{2} \sum \gamma_{\omega}^{\text {out }}\left(a_{\vec{p}}^{\text {in } \dagger}\right)^{2}} \mid \text { in }>
$$

where

$$
\gamma_{\omega}^{\text {out }}=-\frac{\beta_{\vec{p}}^{*}}{\alpha_{\vec{p}}^{*}}=\frac{\lambda^{-2 i \omega} \Gamma(1+2 i \omega)}{\Gamma(1-2 i \omega)} e^{-2 \pi \omega} .
$$

The magnitude of this result will be reproduced for large $\omega$ in our CFT analysis of TBL.

\subsection{Two-Point Function as Stringy Bogolubov Coefficient}

We would like to improve on the minisuperspace analysis and obtain exact CFT results. Our first order of business is to understand what correlator or other quantity in the CFT determines the open string production rate. In order to understand this, we first review certain aspects of the spacelike boundary Liouville (SBL) theory with action (1.5). Quantum states can be described as an incoming wave $e^{i p \phi}$ from the free region $\phi \rightarrow-\infty$ which reflects off the exponential $V \sim e^{b \phi}$ potential and returns as an outgoing 
wave $d_{b}(p) e^{-i p \phi}$, where the reflection coefficient $d_{b}(p)$ is a phase. The state then has the zero mode dependence in the free region

$$
\phi \rightarrow-\infty, \quad \Psi_{p}(\phi) \rightarrow e^{-i p \phi}+R_{b}(-i p) e^{i p \phi} .
$$

Under the barrier, roughly speaking the WKB wave function (for normalizable states) dies exponentially as $\exp (-\sqrt{V}) \sim \exp \left(-e^{b \phi / 2}\right)$, though of course the theory is strongly coupled in this region so that statement is heuristic. According to the operator state correspondence, the reflection coefficient is given by

$$
R_{b}(-i p)=d_{b}\left(\frac{Q}{2}-i p\right)
$$

where $Q=b+\frac{1}{b}$ and the two-point boundary correlator on the upper half plane is

$$
d_{b}(\alpha)=<e^{\alpha \phi} e^{\alpha \phi}>_{S B L}
$$

where the insertions are at $z=0$ and $z=1$. This is illustrated in figure 1a.

Let us now consider analytic continuation $\phi \rightarrow-i X$ from SBL to TBL, so that $X$ is now a timelike target space coordinate, and $p \rightarrow i \omega$, as shown in figure $1 \mathrm{~b}$. There is also an analytic continuation of the screening charge such that $b \rightarrow i, \quad Q \rightarrow 0$ which will be detailed below. Then in the free region $e^{i p \phi} \rightarrow e^{i \omega X}$, and the wave function behaves ast

$$
X \rightarrow-\infty, \quad \Psi_{\omega}(X) \rightarrow e^{-i \omega X}+d_{i}(\omega) e^{i \omega X}
$$

where the appropriately continued reflection coefficient

$$
d_{i}(\omega)=<e^{-i \omega X} e^{-i \omega X}>_{T B L}
$$

is no longer necessarily a pure phase. In the strong coupling region, the potential is now negative relative to the kinetic term, and the wave function behaves as $\exp (-\sqrt{V}) \sim$

\footnotetext{
7 Since $Q \rightarrow 0$ we need not distinguish between $R_{b}$ and $d_{b}$ here.
} 


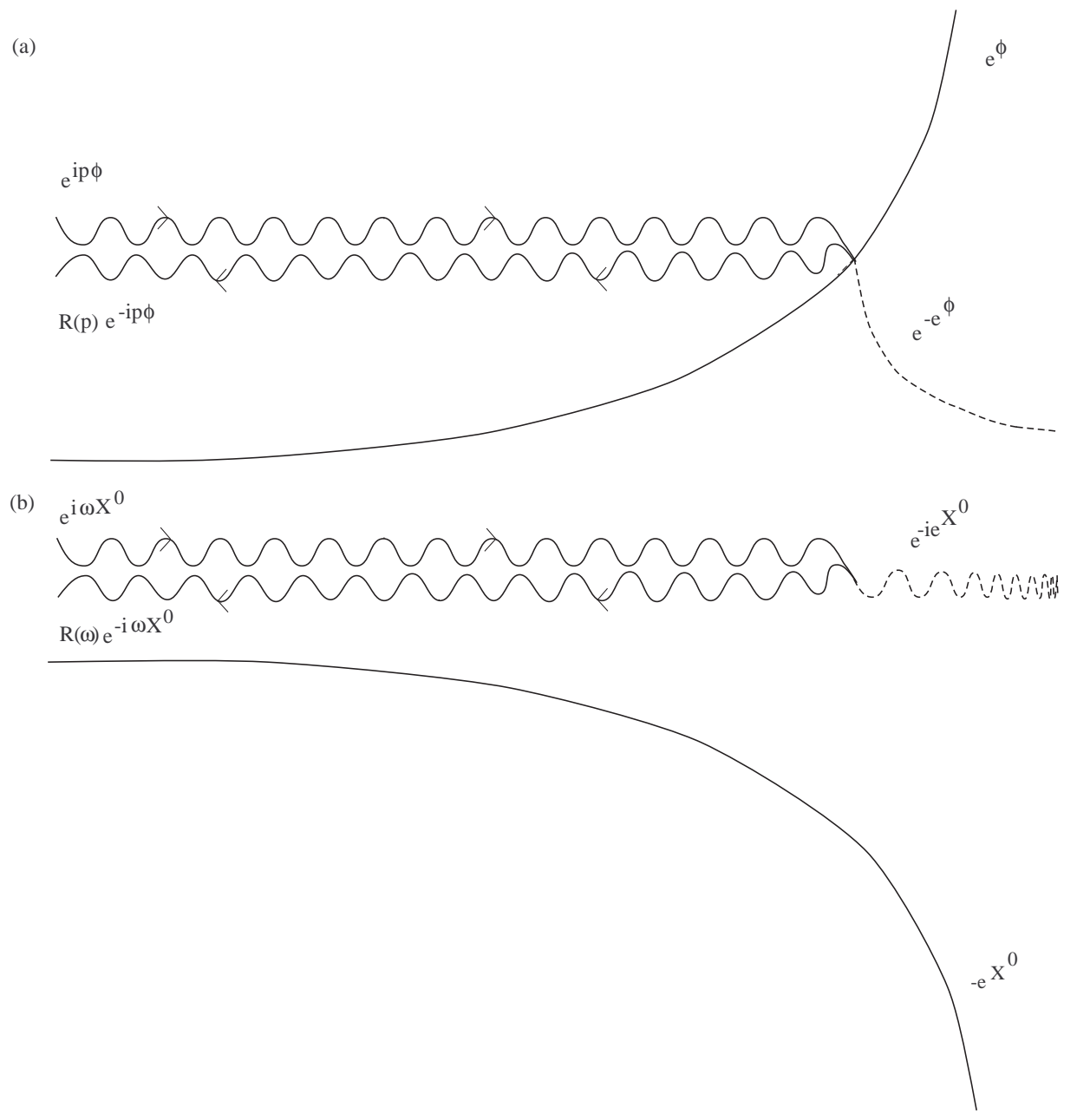

Fig. 1: (a) reflection amplitude for spacelike boundary Liouville and (b) analytically continued amplitude for TBL.

$\exp \left( \pm i e^{X / 2}\right)$ (as indicated in the minisuperspace result (2.4)). In the spacelike case, there are also two possible exponential behaviors (growth and decay) but normalizability singles out the decaying mode. Hence analytic continuation from SBL to TBL yields a wave function with only one of the two asymptotic behaviors, as opposed to a general admixture. Let us choose our prescription so that this is the positive frequency outgoing wave. Then we may interpret (2.15) as the linear combination of incoming waves that produces a purely positive frequency outgoing wave. Comparing with (2.6) we then see that the two-point 
function is a ratio of Bogolubov coefficients

$$
d_{i}(\omega)=\frac{\beta_{\omega}}{\alpha_{\omega}}
$$

The string pair production rate is determined by the magnitude of $d$. In the minisuperspace approximation

$$
\left|d_{i}(\omega)\right|=e^{-2 \pi \omega} .
$$

There is another way of understanding the relation (2.17). Mathematically, correlators in TBL are not unique because one must specify an analytic continuation procedure. Physically they are not unique because one must specify the vacuum state in a time-dependent background. As discussed above, analytic continuation from SBL to TBL most naturally gives correlators in the out vacuum. The out vacuum is represented as an excited state of the in vacuum in expression (2.10). One then easily finds that, in this state, the minisuperspace S-matrix for scattering two incoming strings to zero outgoing strings is given by

$$
-\gamma_{\omega}^{\text {out } *}=\frac{\beta_{\omega}}{\alpha_{\omega}}=d_{i}(\omega)
$$

Hence one may interpret the two point function (2.16) as giving this S-matrix element.

It is natural to conjecture that this relationship extends beyond the example discussed here. More generally we expect that the disk or sphere two-point function for open or closed strings in a time dependent background gives the stringy Bogolubov coefficients.

Now we turn to computation of the CFT correlators.

\section{Bulk Correlators}

The TBL theory is a boundary deformation of a free timelike boson on the upper half plane

$$
S_{T B L}=-\frac{1}{2 \pi} \int_{\Sigma} \partial X \bar{\partial} X+\frac{\lambda}{2} \int_{\partial \Sigma} e^{X}
$$


A correlator involving $n$ closed string vertices and $m$ open string vertices is formally given by the path integral

$$
\begin{aligned}
A & =\left\langle\prod_{i=1}^{n} e^{\beta_{i} X\left(z_{i}, \bar{z}_{i}\right)} \prod_{j=1}^{m} e^{\gamma_{j} X\left(x_{j}\right)}\right\rangle \\
& =\int D X e^{-S} \prod_{i=1}^{n} e^{\beta_{i} X\left(z_{i}, \bar{z}_{i}\right)} \prod_{j=1}^{m} e^{\gamma_{j} X\left(x_{j}\right)}
\end{aligned}
$$

where $x_{i}$ is a point on the real axis. Following [24], we decompose $X=q+\hat{X}$ where $q$ is the zero mode of $X$. Then the $q$ integral can be done exactly, yielding

$$
A=\Gamma(-s)\left(\frac{\lambda}{2}\right)^{s}\left\langle\prod_{i=1}^{n} e^{\beta_{i} \hat{X}\left(z_{i}, \bar{z}_{i}\right)} \prod_{j=1}^{m} e^{\gamma_{j} \hat{X}\left(x_{j}\right)}\left(\int d y e^{\hat{X}(y)}\right)^{s}\right\rangle_{F r e e}
$$

where $s=-\sum_{j}^{n} \beta_{j}-\sum_{i}^{m} \gamma_{i}$. For integer $s, \Gamma(-s)$ has a simple pole and the residue is given by the integral over the nonzero modes, which can be evaluated using the free field correlation function on the half plane with Neumann boundary conditions. (A useful reference for the resulting integrals is [25]). The general correlator is then obtained by analytic continuation in $s$. However, since the residues can be perturbatively evaluated only for integer $s$, and analytic continuation from the integers in not unique, the final answer must be checked using various consistency conditions such as factorization, crossing symmetry, etc. This technique was used in the calculation of the three point function for the bulk Liouville theory [26] [27].

This procedure however is a bit problematic for open string correlators because of singularities when the boundary operator and interaction insertions coincide. A prescription must be specified for dealing with these correlators. Ultimately we believe that this corresponds to the ambiguity in the choice of open string vacuum, to which closed string correlators on the disk are insensitive.

Rather than directly computing the integrals in (3.3), bulk correlators of the form (3.2) may alternately be evaluated using contour deformation. TBL has a level one SU(2) 
current algebra generated by 8

$$
j_{ \pm}(z)=e^{ \pm X(z)}, \quad j_{3}(z)=\frac{1}{2} \partial X(z)
$$

which obey

$$
\begin{aligned}
j_{-}(z) j_{+}(w) & \sim \frac{1}{(z-w)^{2}}-\frac{2 j_{3}(w)}{(z-w)} \\
j_{3}(z) j_{ \pm}(w) & \sim \pm \frac{j_{ \pm}(w)}{(z-w)}
\end{aligned}
$$

Note however that in the standard norm for a timelike boson $X, j_{3}$ is anti-hermitian while $j^{ \pm}$are both hermitian. Nevertheless the charges

$$
J_{ \pm}=\oint \frac{d z}{2 \pi i} j_{ \pm}(z), \quad J_{3}=\oint \frac{d z}{2 \pi i} j_{3}(z)
$$

obey the usual commutation relations

$$
\left[J_{-}, J_{+}\right]=-2 J_{3}, \quad\left[J_{3}, J_{ \pm}\right]= \pm J_{ \pm}
$$

and are therefore useful for computing correlators. To exploit this we note that the TBL boundary interaction is simply

$$
\frac{\lambda}{2} \int_{\partial \Sigma} d \tau e^{X}=i \pi \lambda J^{+}
$$

Correlators may then be evaluated by e.g. deforming the $J^{+}$contour into the upper half plane and letting it act on the operator insertions as in [5].9

\subsection{One-Point Function}

In this section we calculate the one-point function of the closed string vertex operator $e^{-n X}$ inserted at the center of a unit disk. Because of momentum conservation the

\footnotetext{
8 In our conventions $X(z, \bar{z})=\frac{1}{2}(X(z)+X(\bar{z})), X(z) X(w) \sim 2 \ln (z-w)$ and $\alpha^{\prime}=1$.

9 Life is not so simple with boundary insertions because one must specify the contour prescription near the boundary operator insertion.
} 
perturbative contribution is given by the insertion of $n$ Liouville boundary interaction terms.

$$
\begin{aligned}
\left\langle e^{-n X}(z, \bar{z})\right\rangle_{T B L} & =\left(\frac{\lambda}{2}\right)^{n}\left\langle e^{-n X}(z, \bar{z}) \prod_{i=1}^{n} \int d x_{i} e^{X}\left(x_{i}\right)\right\rangle \\
& =\left(\frac{\lambda}{2}\right)^{n}|z-\bar{z}|^{\frac{n^{2}}{2}} \prod_{i=1}^{n} \int d x_{i} \prod_{i<j}\left|x_{i}-x_{j}\right|^{2} \prod_{i=1}^{n}\left|z-x_{i}\right|^{-n}\left|\bar{z}-x_{i}\right|^{-n} \\
& =\left(\frac{\lambda}{2}\right)^{n}|z-\bar{z}|^{-\frac{n^{2}}{2}} \int \prod_{i=1}^{s} \frac{d u_{i}}{u_{i}} \prod_{i<j}^{s}\left|u_{i}-u_{j}\right|^{2} \\
& =|z-\bar{z}|^{-\frac{n^{2}}{2}}\left(\frac{\lambda}{2}\right)^{n}(2 \pi)^{n} \Gamma(n+1) .
\end{aligned}
$$

Where is the third line the upper half plane was mapped to the disk, which mapped the integrations to the well known Dyson-gas form. The perturbative result (3.9) can now be used to determine the general form of the bulk one-point function by analytical continuation using (3.3).

$$
\begin{aligned}
\left\langle e^{\beta X}(z, \bar{z})\right\rangle_{T B L} & =|z-\bar{z}|^{-2 h_{\beta}}(\pi \lambda)^{-\beta} \Gamma(\beta) \Gamma(1-\beta) \\
& =|z-\bar{z}|^{-2 h_{\beta}}(\pi \lambda)^{-\beta} \frac{\pi}{\sin \pi \beta}
\end{aligned}
$$

The one-point function (3.10) can now be analytically continued via $\beta \rightarrow-i \omega$, giving

$$
\left\langle e^{-i \omega X}(z, \bar{z})\right\rangle_{T B L}=|z-\bar{z}|^{-2 h_{\omega}}(\pi \lambda)^{i \omega} \frac{\pi}{i} \frac{1}{\sinh \pi \omega} .
$$

\subsection{Boundary States}

The collection of all bulk one-point functions can be represented by a boundary state. The boundary state $|B\rangle_{B S G}$ for the boundary Sine-Gordon theory

$$
S=\frac{1}{2 \pi} \int_{\Sigma} \partial \phi \bar{\partial} \phi+\frac{1}{2} \int_{\partial \Sigma}\left(\lambda e^{i} \phi+\bar{\lambda} e^{-i \phi}\right)
$$

was found using the bulk $S U(2)$ current algebra in [5](see also [6,28, 229,30]). For a noncompact boson one has (up to normalization)

$$
\left.|B\rangle_{B S G}=\sum_{j} \sum_{m=-j}^{j} D_{m,-m}^{j}(g(\lambda, \bar{\lambda}))|j ; m, m\rangle\right\rangle .
$$


Here $|j ; m, m\rangle\rangle$ is the Ishibashi state associated with the $S U(2)$ primary field $|j ; m, m\rangle$. $D_{m,-m}^{j}(g)$ is the spin $j$ representation of the $S U(2)$ rotation given by

$$
g(\lambda, \bar{\lambda})=e^{i \pi\left(\lambda J_{+}+\bar{\lambda} J_{-}\right)}=\left(\begin{array}{cc}
\cos (\pi|\lambda|) & i \lambda \frac{\sin (\pi|\lambda|)}{|\lambda|} \\
i \bar{\lambda} \frac{\sin (\pi|\lambda|)}{|\lambda|} & \cos (\pi|\lambda|)
\end{array}\right) .
$$

Reality of the boundary interaction demands that $\lambda$ and $\bar{\lambda}$ are complex conjugates. Sen [14, 15] observed that an analytic continuation $\phi \rightarrow-i X^{0}$ produces an exact time dependent open string background.

It was pointed out in [3] (and at intermediate stages of the calculations in [5]) that the boundary states can also be constructed for $g \in S L(2, C)$, and in particular we can set $\bar{\lambda}=0$ to obtain the non-hermitian theory whose action $S_{N H}$ is in (1.4). The unitary rotation matrix (3.14) becomes a raising operator. The boundary state becomes simply

$$
\left.|B\rangle_{N H}=\sum_{j} \sum_{m \geq 0}^{j}\left(\begin{array}{c}
j+m \\
2 m
\end{array}\right)(i \pi \lambda)^{2 m}|j ; m, m\rangle\right\rangle .
$$

Inspection of the $S U(2)$ currents (3.4) of TBL theory reveals that under $\phi \rightarrow-i X^{0}$, $J_{k} \rightarrow J_{k}$ and hence $\left.\left.|j ; m, m\rangle\right\rangle \rightarrow|j ; m, m\rangle\right\rangle$. Therefore we may also write

$$
\left.|B\rangle_{T B L}=\sum_{j} \sum_{m \geq 0}^{j}\left(\begin{array}{c}
j+m \\
2 m
\end{array}\right)(i \pi \lambda)^{2 m}|j ; m, m\rangle\right\rangle .
$$

Following a related discussion in [14], the component of the boundary state (3.16) which does not contain any oscillator modes can be obtained by setting $m=j$ : 10

$$
\begin{aligned}
|B\rangle_{0} & =\sum_{j}(i \pi \lambda)^{2 j}|j ; j, j\rangle \\
& =\sum_{n=0}^{\infty}(-\pi \lambda)^{n} e^{n q}|0\rangle \\
& =\frac{1}{1+\lambda \pi e^{q}}|0\rangle .
\end{aligned}
$$

10 As in [14 there are extra phases $j^{j}$ appearing in $|j ; j, j\rangle$ which can be fixed by demanding that the $\lambda=\bar{\lambda}=1 / 2$ state corresponds to an array of D0-branes. 
This result agrees with the appropriate limit of the more general boundary state found in [14]. Although every term in the second line of (3.17) diverges at late times the resummed expression is well behaved, in particular there exists a Fourier transform which gives the closed string one-point function

$$
\left\langle e^{2 i \omega X} \mid B\right\rangle=\operatorname{const}(\pi \lambda)^{2 i \omega} \frac{1}{\sinh (2 \pi \omega)},
$$

in agreement with (3.11).

\subsection{N-Point Correlators}

Perturbative correlation functions involving only bulk vertex operators can be easily evaluated using contour deformation techniques. For example the two-point function is

$$
\begin{aligned}
A_{2}\left(j_{1}, j_{2}\right) & =\left\langle e^{-2 j_{1} X}\left(z_{1}, \bar{z}_{1}\right) e^{-2 j_{2} X}\left(z_{2}, \bar{z}_{2}\right)\right\rangle_{T B L} \\
& =(2 \pi i)^{2\left(j_{1}+j_{2}\right)}\left(\frac{\lambda}{2}\right)^{2\left(j_{1}+j_{2}\right)} \frac{1}{\left(2\left(j_{1}+j_{2}\right)\right) !} \\
& \times\left\langle e^{-j_{1} X}\left(z_{1}\right) e^{-j_{1} X}\left(\bar{z}_{1}\right) e^{-j_{2} X}\left(z_{2}\right) e^{-j_{2} X}\left(\bar{z}_{2}\right) \prod_{i=1}^{2\left(j_{1}+j_{2}\right)} \oint \frac{d x_{i}}{2 \pi i} e^{X}\left(x_{i}\right)\right\rangle \\
& =(2 \pi i)^{2\left(j_{1}+j_{2}\right)}\left(\frac{\lambda}{2}\right)^{2\left(j_{1}+j_{2}\right)}\left\langle e^{-j_{1} X}\left(z_{1}\right) e^{+j_{1} X}\left(\bar{z}_{1}\right) e^{-j_{2} X}\left(z_{2}\right) e^{+j_{2} X}\left(\bar{z}_{2}\right)\right\rangle \\
& =(2 \pi i)^{2\left(j_{1}+j_{2}\right)}\left(\frac{\lambda}{2}\right)^{2\left(j_{1}+j_{2}\right)}\left|z_{1}-\bar{z}_{1}\right|^{-\frac{j_{1}^{2}}{2}}\left|z_{2}-\bar{z}_{2}\right|^{-\frac{j_{2}^{2}}{2}}\left|z_{1}-z_{2}\right|^{j_{1} j_{2}}\left|z_{1}-\bar{z}_{2}\right|^{-j_{1} j_{2}}
\end{aligned}
$$

In the second line the bulk vertex operators on the half plane where split into holomorphic and antiholomorphic parts on the plane using the doubling trick. Then the contours along the real axis were pulled off the lower half plane and the $S U(2)$ algebra (3.7) was used to turn $e^{-j_{i} X}\left(\bar{z}_{i}\right)$ into $e^{+j_{i} X}\left(\bar{z}_{i}\right)$. Note that all combinatorial factors cancel in the end. It is straightforward to generalize the contour deformation techniques to evaluate bulk N-point functions. Hence as far as the bulk correlation functions are concerned the TBL theory is very simple. We shall see that this is not the case for correlation functions involving boundary vertex operators. 


\section{Boundary Correlators}

As mentioned above, the simple methods for computing bulk correlators encounter ambiguous singularities when applied to boundary correlators. In this section we will define the two-point correlator by analytic continuation from known expressions for the two-point correlator of the spatial boundary Liouville theory [8,9]. We shall see that even this procedure is ambiguous: an infinite number of pairs of poles and singularities accumulate at precisely the point we wish to continue to. We will adopt a simple (but not

obviously unique) prescription in which these poles and singularities cancel one another and a finite answer is obtained for the two-point correlator.

\subsection{Spacelike Boundary Liouville}

Spacelike boundary Liouville theory can be defined by the following action on the half plane:

$$
S_{S B L}=\frac{1}{2 \pi} \int_{\Sigma}\left(\partial \phi \bar{\partial} \phi+\pi \mu e^{2 b \phi}\right)+\frac{\lambda}{2} \int_{\partial \Sigma} e^{b \phi} .
$$

Here $\mu$ and $\lambda$ are the bulk and boundary cosmological constants respectively. The Liouville coupling constant $b$ determines the background charge $Q=b+\frac{1}{b}$ and the central charge $c=1+6 Q^{2}$ of the theory. Boundary vertex operators $e^{\omega \phi}$ have conformal dimension $h_{\omega}=\omega(Q-\omega)$. There are two important quantities calculated by Fateev, Zamolodchikov and Zamolodchikov [8](see also [9]). Firstly the bulk one-point function

$$
\begin{aligned}
U(\alpha) & =(z-\bar{z})^{2 h_{\alpha}}\left\langle e^{2 \alpha X(z, \bar{z})}\right\rangle \\
& =\frac{2}{b}\left(\pi \mu \gamma\left(b^{2}\right)\right)^{\frac{Q-2 \alpha}{2 b}} \Gamma\left(2 b \alpha-b^{2}\right) \Gamma\left(\frac{2 \alpha}{b}-\frac{1}{b^{2}}-1\right) \cosh ((Q-2 \alpha) \pi s),
\end{aligned}
$$

where $\gamma(x)=\Gamma(x) / \Gamma(1-x)$. Secondly the boundary two-point function

$$
\left\langle e^{\omega_{1} \phi(x)} e^{\omega_{2} \phi(0)}\right\rangle=\frac{1}{|x|^{2 h_{\omega}}}\left(\delta\left(Q-\omega_{1}-\omega_{2}\right)+\delta\left(\omega_{1}-\omega_{2}\right) d(\omega)\right)
$$

where

$$
d(\omega)=\left(\pi \mu \gamma\left(b^{2}\right) b^{2-2 b^{2}}\right)^{\frac{Q-2 \omega}{2 b}} \frac{G_{b}(Q-2 \omega)}{G_{b}(2 \omega-Q)} \frac{1}{S_{b}(\omega+i s) S_{b}(\omega-i s) S_{b}(\omega)^{2}}
$$


Here $G_{b}$ and $S_{b}$ are special functions defined in [8] and related to the Barnes double Gamma function [31] (see Appendix A for a collection of useful formulae). The parameter $s$ is related to the coupling constants in (4.1) of the theory in the following way

$$
\cosh ^{2}(\pi b s)=\frac{\lambda^{2}}{4 \mu} \sin \left(\pi b^{2}\right)
$$

Our current interest is the case for which the bulk cosmological constant is turned off. From (4.5) it follows that as $\mu \rightarrow 0$ one has to take $s \rightarrow \infty$.

$$
\lim _{s \rightarrow \infty} U(\alpha)=\frac{1}{b}\left(\frac{\pi \lambda}{\Gamma\left(1-b^{2}\right)}\right)^{\frac{Q-2 \alpha}{b}} \Gamma\left(2 b \alpha-b^{2}\right) \Gamma\left(\frac{2 \alpha}{b}-\frac{1}{b^{2}}-1\right) .
$$

Using (4.5) and A.11 the two-point function has the limit 11

$$
\lim _{s \rightarrow \infty} d(\omega) \equiv d_{b}(\omega)=\left(\frac{\pi \lambda b^{1-b^{2}}}{\Gamma\left(1-b^{2}\right)}\right)^{\frac{Q-2 \omega}{b}} \frac{G_{b}(Q-2 \omega)}{G_{b}(2 \omega-Q)} \frac{1}{S_{b}(\omega)^{2}} .
$$

The SBL theory with interaction $e^{b \phi}$ can (at least formally) be related to the TBL theory with interaction $e^{X}$ by taking $b \rightarrow i$ while $\phi \rightarrow-i X$. Note that in this limit $Q \rightarrow 0, c \rightarrow 1$ and one gets a free boson with vanishing background charge. Furthermore perturbative correlation functions are clearly identical for the two theories.

For the bulk one-point function (4.6) one finds

$$
\lim _{b \rightarrow i} \lim _{s \rightarrow \infty} U(\alpha)=\pi(\pi \lambda)^{2 i \alpha} \frac{1}{\sinh (2 \pi \alpha)},
$$

which (up to normalization) agrees with (3.18) for $\alpha=\omega$.

\subsection{Analytic Continuation to Timelike Boundary Liouville}

We wish to obtain the TBL two-point function from the SBL two point function (4.4) by the analytic continuation

$$
\left\langle e^{-i \omega X} e^{-i \omega X}\right\rangle_{T B L} \equiv\left\langle e^{\omega \phi} e^{\omega \phi}\right\rangle_{S B L, b=i}=d_{i}(\omega) .
$$

11 This formula appears in [7], but apparently with a different power of 2 in the normalization. 
This however is not as straightforward as it sounds. As seen in the appendix, the special functions $G_{b}(z)$ and $S_{b}(z)$ appearing in (4.4) have poles and/or zeros at $z=m b+\frac{n}{b}$ for integer $m$ and $n$. If we take $b \rightarrow i$ from the real axis along the unit circle these poles/zeros are at $z=(m+n) \operatorname{Re} b+(m-n) \operatorname{Im} b$, and an infinite number of them accumulate at every integer multiple of $i .12$ For this reason $G_{b}$ is not defined for $b=i$ 31].

However it turns out that if we look at the particular ratio of special functions appearing in $d_{b}(\omega)(4.7)$, we shall see that the poles and singularities accumulate in pairs and can be arranged to cancel for real $\omega$. This will enable us to give a prescription defining $d_{b}$. Using recursion and product relations from the appendix, the ratio of special functions appearing in $d_{b}$ is

$$
\begin{aligned}
\frac{G_{b}(Q-2 \omega)}{G_{b}(2 \omega-Q)} \frac{1}{S_{b}(\omega)^{2}} \\
\quad=\frac{G_{b}(Q-2 \omega)}{G_{b}(2 \omega-Q)} \frac{G_{b}(\omega)^{2}}{G_{b}(Q-\omega)^{2}} \\
\quad=Y_{b}(\omega) b^{\frac{2 \omega}{b}-2 b \omega-\frac{1}{b^{2}}+b^{2}} \frac{\Gamma\left(\frac{2 \omega}{b}-\frac{1}{b^{2}}\right) \Gamma\left(2 \omega b-1-b^{2}\right) \Gamma\left(-\frac{2 \omega}{b}+1\right) \Gamma(-2 \omega b)}{\Gamma^{2}\left(-\frac{\omega}{b}+1\right) \Gamma^{2}(-b \omega)},
\end{aligned}
$$

where

$$
Y_{b}(\omega) \equiv \frac{G_{b}(-2 \omega)}{G_{b}(2 \omega)} \frac{G_{b}(\omega)^{2}}{G_{b}(-\omega)^{2}} .
$$

Using the product representation A.10 one has simply

$$
Y(\omega)=\prod_{m=0}^{\infty} \prod_{n=0}^{\infty}\left(\frac{2 \omega+\Omega}{-2 \omega+\Omega}\right)\left(\frac{-\omega+\Omega}{\omega+\Omega}\right)^{2} .
$$

where $\Omega=m b+n / b$. It can be seen that the product is absolutely convergent for generic complex $b$.

We now wish to understand the behavior of this correlator for $b \rightarrow i$. We will take $b \rightarrow i$ by first going to the imaginary axis, so that $b=i \beta$ and $Q=i\left(\beta-\frac{1}{\beta}\right)$ with $\beta$ real, and then taking $\beta \rightarrow 1$. Physically this corresponds to adding a real timelike linear dilaton

12 This singularity may be related to the accumulation of boundary minimal models at $c=1$ 13, 14. 
which alters the growth of the tachyon. For pure imaginary $b, \Omega$ is also pure imaginary, and (for real $\omega$ ) the factors in (4.12) appear in complex conjugate pairs. Hence for this case $Y$ is formally a pure phase. In order to make a more precise statement and determine the phase we now introduce the integral form of the special functions.

In [8] one finds the integral representation

$$
\ln S_{b}(x)=\frac{1}{2} \int_{0}^{\infty} \frac{d t}{t}\left[\frac{\sinh (Q-2 x) t}{\sinh (b t) \sinh (t / b)}+\frac{2 x-Q}{t}\right] .
$$

We take $\operatorname{Im} b>0, \operatorname{Re} b>0$ and $0<2 x<Q$ with $x$ real, which implies convergence of (4.13). Other values of the parameters will be obtained by analytic continuation. By deforming the integration contour, (4.13) may be rewritten

$$
\ln S_{b}(x)=I_{b}(x)+\Sigma_{b}(x)
$$

as the sum of an integral $I_{b}(x)$ over the positive imaginary axis plus a sum $\Sigma_{b}(x)$ of simple pole residues at $t=\frac{n \pi i}{b} .13,14$ (The contribution from the quarter-circle at infinity vanishes.) Defining $t=i \tau$ the integral is

$$
I_{b}(x)=\frac{i}{2} \int_{0}^{\infty} \frac{d \tau}{\tau}\left[\frac{\sin ((2 x-Q) \tau)}{\sin (b \tau) \sin (\tau / b)}+\frac{Q-2 x}{\tau}\right] .
$$

For $b \rightarrow i \beta$ with $\beta$ real and positive, this reduces to the convergent expression

$$
I_{i \beta}(x)=\frac{i}{2} \int_{0}^{\infty} \frac{d \tau}{\tau}\left[\frac{\sin ((2 x-Q) \tau)}{\sinh (\beta \tau) \sinh (\tau / \beta)}+\frac{Q-2 x}{\tau}\right]
$$

For $b \rightarrow i$, this further reduces to

$$
I_{i}(x)=\frac{i}{2} \int_{0}^{\infty} \frac{d \tau}{\tau}\left[\frac{\sin (2 x \tau)}{\sinh ^{2}(\tau)}-\frac{2 x}{\tau}\right],
$$

13 We might also have deformed to the negative imaginary axis which would have picked up the poles at $t=-n \pi i b$ and changed the results below by the replacement $b \rightarrow \frac{1}{b}$. One possibility is to take half the sum of the two contours which would manifestly preserve the $b \rightarrow \frac{1}{b}$ symmetry. However there is no change in the final formulae for $b=i$, which is our main interest here, so we will not further explore these alternate prescriptions.

14 In the appendix of [4] it was suggested that the residue sum might be dropped in determining the correlators. That leads to correlators which are pure phases of constant magnitude. 
which contributes a pure phase to $S$. The sum over pole residues is

$$
\begin{aligned}
\Sigma_{b}(x) & =\sum_{n=1}^{\infty} \frac{(-)^{n} \sin \left(\frac{\pi n(Q-2 x)}{b}\right)}{n \sin \left(\frac{\pi n}{b^{2}}\right)} \\
& =\sum_{n=1}^{\infty} \frac{1}{n}\left[\cos \left(\frac{2 \pi n x}{b}\right)-\cot \left(\frac{\pi n}{b^{2}}\right) \sin \left(\frac{2 \pi n x}{b}\right)\right] .
\end{aligned}
$$

In order to take $b \rightarrow i \beta$ in this expression, we define

$$
\frac{1}{b^{2}}=-\frac{1}{\beta^{2}}+i \epsilon, \quad x=-i b y
$$

where $y$ is real.15 The real part of the sum is then

$$
\operatorname{Re}\left[\Sigma_{i \beta, \epsilon}\right]=\sum_{n=1}^{\infty} \frac{1}{n}\left[\cosh (2 \pi n y)+\sinh (2 \pi n y) \operatorname{Im}\left[\cot \left(\frac{n \pi}{\beta^{2}}-n \pi i \epsilon\right)\right]\right] .
$$

We wish to take $\epsilon \rightarrow 0$ with $y$ fixed, which takes us outside the radius of convergence of (4.20). The problematic terms for small $\epsilon$ are the ones that behave as $e^{2 \pi n y}\left(e^{-2 \pi n y}\right)$ for positive (negative) $y$, ie. the first (second) term in the expression

$$
\operatorname{Re}\left[\Sigma_{i \beta, \epsilon}\right]=\sum_{n=1}^{\infty} \frac{e^{2 \pi n y}}{2 n}\left[1+\operatorname{Im}\left[\cot \left(\frac{n \pi}{\beta^{2}}-n \pi i \epsilon\right)\right]\right]+\sum_{n=1}^{\infty} \frac{e^{-2 \pi n y}}{2 n}\left[1-\operatorname{Im}\left[\cot \left(\frac{n \pi}{\beta^{2}}-n \pi i \epsilon\right)\right]\right] .
$$

When $\epsilon \rightarrow 0$, we will define the first (second) term for positive (negative) real $y$ by analytic continuation from negative (positive) real $y$. The resulting expression is related by analytic continuation to those obtained in the $\epsilon \neq 0$ region where the sum is convergent.

The dangerous-looking term in expression (4.21) for $\epsilon \rightarrow 0$ can be rewritten

$$
\operatorname{Im}\left[\cot \left(\frac{n \pi}{\beta^{2}}-n \pi i \epsilon\right)\right]=\frac{1}{2} \frac{\sinh (2 n \pi \epsilon)}{\sinh ^{2}(n \pi \epsilon)+\sin ^{2}\left(\frac{n \pi}{\beta^{2}}\right)} .
$$

To define the limit we must take $\beta^{2}$ irrational so that $\sin \left(\frac{n \pi}{\beta^{2}}\right)$ is nonzero for every $n$. In that case, the $\sin ^{2}\left(\frac{n \pi}{\beta}\right)$ dominates over the $\sinh ^{2}(n \pi \epsilon)$ term for $\epsilon \rightarrow 0$. Because of the $\sinh (2 n \pi \epsilon)$ in the numerator every term in the sum vanishes for $\epsilon=0$ and hence

$$
\lim _{\epsilon \rightarrow 0} \operatorname{Im}\left[\cot \left(\frac{n \pi}{\beta^{2}}-n \pi i \epsilon\right)\right]=0 .
$$

15 For $b$ on the imaginary axis, reality of $x$ and reality of $y$ are the same thing. Keeping $y$ rather than $x$ real for $b$ off the imaginary axis simplifies the calculations. 
This leaves us with for $\epsilon \rightarrow 0$

$$
\operatorname{Re}\left[\Sigma_{i \beta}\right]=\sum_{n=1}^{\infty} \frac{e^{2 \pi n y}}{2 n}+\sum_{n=1}^{\infty} \frac{e^{-2 \pi n y}}{2 n} .
$$

Using analytic continuation in $y$ to define the sums, and restoring $x=\beta y$ gives

$$
\operatorname{Re}\left[\Sigma_{i \beta}\right]=-\ln \left[2\left(\sinh \frac{\pi x}{\beta}\right)\right] .
$$

Although derived for irrational $\beta^{2}$, this result can obviously be smoothly extended back to the reals. For $\beta \rightarrow 1$, the integral (4.17) is real, and (4.25) is the only real part of $\ln S_{i}$. This then yields for $b \rightarrow i$

$$
\operatorname{Re} \ln \left[S_{i}(x)\right]=-\ln [2 \sinh (\pi x)]
$$

Now we consider the imaginary part of the sum. Here it is useful to consider $\beta^{2}=\frac{q}{p}$ rational (with $(p, q)$ coprime). The imaginary part is then

$$
\operatorname{Im}\left[\Sigma_{i \beta, \epsilon}\right]=-\sum_{n=1}^{\infty} \frac{1}{n} \sinh (2 \pi n y) \operatorname{Re}\left[\cot \left(n \pi \frac{p}{q}-n \pi i \epsilon\right)\right],
$$

where

$$
\operatorname{Re}\left[\cot \left(n \pi \frac{p}{q}-n \pi i \epsilon\right)\right]=\frac{1}{2} \frac{\sin \left(\frac{2 n p \pi}{q}\right)}{\sinh ^{2}(n \pi \epsilon)+\sin ^{2}\left(\frac{n p \pi}{q}\right)} .
$$

Now we find that the terms with $n$ a multiple of $q$ vanish, while the remaining terms are bounded but typically nonvanishing for $\epsilon \rightarrow 0$. The sum is then for $\epsilon=0$

$$
\operatorname{Im}\left[\Sigma_{i \beta}\right]=-\sum_{n \neq m q}^{\infty} \frac{1}{n} \sinh (2 \pi n y) \cot \left(n \pi \frac{p}{q}\right) .
$$

Again we will define the $e^{2 \pi n y}$ terms by analytic continuation from negative $y$ but for the sake of brevity we will not bother to separate the two types of terms. Writing $n=m q+n_{0}$, with $n_{0}=1, \ldots(q-1),(4.29)$ may be rewritten

$$
\begin{aligned}
\operatorname{Im}\left[\Sigma_{i \beta}\right] & =-\sum_{n_{0}=1}^{q-1} \sum_{m=0}^{\infty} \frac{\sinh \left(2 \pi\left(m q+n_{0}\right) \frac{x}{\beta}\right)}{m q+n_{0}} \cot \left(n_{0} \pi \frac{p}{q}\right) \\
& =\int_{\frac{-x}{\beta}}^{\frac{x}{\beta}} d y \frac{\pi}{e^{-2 \pi q y}-1} \sum_{n_{0}=1}^{q-1} \cot \left(n_{0} \pi \frac{p}{q}\right) e^{-2 \pi n_{0} y} .
\end{aligned}
$$


The integral has an unilluminating expression in terms of hypergeometric functions. Note that for the case of current interest $b=i, p=q=1$ and (4.30) trivially vanishes. (4.30) is a finite expression which (together with (4.17)) defines the phase of $S_{b}(x)$ for real $x$ and $b$ on the imaginary axis. It is a smooth function of $x$ for any rational $b$. It is easy to see however 16 , that it is not a smooth function of $\beta^{2}$ : it varies chaotically over the rationals, and has no obvious extension to the reals.

Now let us consider the product $Y$ appearing in (4.12). This can be written in terms of $S_{b}$ as

$$
Y_{b}(\omega)=\frac{S_{b}(2 \omega)}{S_{b}^{2}(\omega)} \frac{b}{2 \pi} \frac{\Gamma^{2}\left(1-\frac{\omega}{b}\right) \Gamma^{2}(-b \omega)}{\Gamma\left(1-\frac{2 \omega}{b}\right) \Gamma(-2 b \omega)} .
$$

It then follows from (4.17), (4.25) and (4.30) that

$$
Y_{i}(\omega)=-e^{i \theta(\omega)}
$$

which is a pure phase in agreement with the naive expectation from the product formula (4.12). The phase is determined by (4.17) as

$$
\theta=-i I_{i}(2 \omega)+2 i I_{i}(\omega)=\frac{1}{2} \int_{0}^{\infty} \frac{d \tau}{\tau} \frac{\sin (4 \omega \tau)-2 \sin (2 \omega \tau)}{\sinh ^{2}(\tau)}
$$

Hence, our continuation prescription yields

$$
d_{i}(\omega)=\frac{(\pi \lambda)^{2 i \omega} e^{i \theta(\omega)}}{4 \cosh ^{2}(\pi \omega)}
$$

This agrees asymptotically for large $\omega$ with the minisuperspace result for the string creation rate $(2.18)$.

It is interesting to consider the results of taking other contours from $\operatorname{Re} b>0$ to $b=i$. Consider for example taking $b \rightarrow i$ along the arc $b=e^{i \theta}$ for $0 \leq \theta \leq \frac{\pi}{2}$, which has real $Q=2 \cos \theta$. In this case the phase of $Y$ is smooth (in fact it vanishes) but the magnitude

16 For example $q=p+1$ for large $p$ does not approach $q=p=1$. 
fluctuates wildly as a function of $\omega$ for $\theta \rightarrow \frac{\pi}{2}$. This can be seen from the recursion relation 17

$$
Y_{b}(\omega+Q)=\frac{4(\omega+Q)}{\omega}\left|\frac{\Gamma(-2 b \omega) \Gamma^{2}(-b(\omega+Q))}{\Gamma(-2 b(\omega+Q)) \Gamma^{2}(-b \omega)}\right|^{2}|\cot (\pi b \omega) \sin \pi b(2 \omega+Q)|^{2} Y_{b}(\omega)
$$

For $b \rightarrow i, Q \rightarrow 0$, and this reduces to

$$
Y_{i}(\omega+0)=16 \cosh ^{4}(\pi \omega) Y_{i}(\omega)
$$

Hence along this contour the two-point function does not smoothly approach (4.34), in accord with the general expectation that correlators at $b=i$ will depend on a contour prescription.

\section{Acknowledgments}

The work of A.S. was supported in part by DOE grant DE-FG02-91ER40654 The work of M.G. was supported by NSF grant 9870115 . We are grateful to I. Affleck, V. Kazakov, M. Kleban, I. Kostov, D. Kutasov, J. Maldacena, A. Maloney, S. Minwalla, D. Nelson, J. Polchinski, A. Sen, S. Shenker, J. Teschner, X. Yin, A. Zamolodchikov and especially V. Schomerus and A. Recknagel for useful discussions. M.G. gratefully acknowledges the hospitality of LPTHE, Paris and especially B. Pioline during the finalization of this paper.

\section{Appendix A. Properties of special functions}

The special function $G_{b}$ was defined in [8] (see also [11]). It obeys the recursion relations

$$
G_{b}(x+b)=(2 \pi)^{-\frac{1}{2}} b^{-b x+\frac{1}{2}} \Gamma(b x) G_{b}(x), \quad G_{b}\left(x+\frac{1}{b}\right)=(2 \pi)^{-\frac{1}{2}} b^{\frac{x}{b}-\frac{1}{2}} \Gamma\left(\frac{x}{b}\right) G_{b}(x) .
$$

There exists an integral representation for $G_{b}(x)$, which is valid for $\operatorname{Re}(x)>0$,

$$
\ln G_{b}(x)=\int_{0}^{\infty} \frac{d t}{t}\left(\frac{e^{-\frac{Q t}{2}}-e^{-x t}}{\left(1-e^{-b t}\right)\left(1-e^{-\frac{t}{b}}\right)}+\frac{\left(\frac{Q}{2}-x\right)^{2}}{2} e^{-t}+\frac{\frac{Q}{2}-x}{t}\right) .
$$

17 Physically the recursion formulae are derived by considering insertions of degenerate operators. 
Another useful special function is defined by $S_{b}(x)=G_{b}(Q-x) / G_{b}(x)$ and satisfies the recursion relations

$$
\begin{aligned}
S_{b}(x+b) & =2 \sin (\pi b x) S_{b}(x), \\
S_{b}\left(x+\frac{1}{b}\right) & =2 \sin \left(\pi \frac{x}{b}\right) S_{b}(x), \\
S_{b}(x+Q) & =-4 \sin (\pi b x) \sin \left(\pi \frac{x}{b}\right) S_{b}(x),
\end{aligned}
$$

as well as

$$
S_{b}(x) S_{b}(-x)=-\frac{1}{4 \sin (\pi b x) \sin \left(\pi \frac{x}{b}\right)} .
$$

Since $Q=b+\frac{1}{b}$ one can easily show by applying (A.1) twice that

$$
\begin{aligned}
& G_{b}(x+Q)=\frac{b^{\frac{x}{b}-b x+1}}{2 \pi} \Gamma\left(\frac{x}{b}+1\right) \Gamma(b x) G_{b}(x), \\
& G_{b}(x-Q)=\frac{b^{-\frac{x}{b}+b x+\frac{1}{b^{2}}-b^{2}-1} 2 \pi}{\Gamma\left(\frac{x}{b}-\frac{1}{b^{2}}\right) \Gamma\left(b x-1-b^{2}\right)} G_{b}(x) .
\end{aligned}
$$

$G_{b}(x)$ is related to a the Barnes double Gamma function $\Gamma_{2}\left(x \mid \nu_{1}, \nu_{2}\right)$ [31] in the following way

$$
G_{b}(z)=\Gamma_{2}^{-1}(z \mid b, 1 / b) .
$$

The Barnes double Gamma function is related to the double Hurwitz function [31,32]

$$
\zeta_{2}^{s}\left(x \mid \nu_{1}, \nu_{2}\right)=\sum_{m, n>0}\left(n \nu_{1}+m \nu_{2}+x\right)^{-s}
$$

in the following way

$$
\Gamma_{2}\left(x \mid \nu_{1}, \nu_{2}\right)=\exp \left(\left.\frac{\partial}{\partial s} \zeta_{2}^{s}\left(x \mid \nu_{1}, \nu_{2}\right)\right|_{s=0}\right) .
$$

It follows from (A.7) and (A.8) that $\Gamma_{2}$ has a product representation

$$
\Gamma_{2}^{-1}\left(z \mid \nu_{1}, \nu_{2}\right)=e^{\frac{z^{2}}{2 \gamma_{21}}+z \gamma_{22}} z \prod_{m=0}^{\infty} \prod_{n=0}^{\infty}\left(1+\frac{z}{\Omega}\right) e^{-\frac{z}{\Omega}+\frac{z^{2}}{2 \Omega^{2}}},
$$

where $\Omega=m \nu_{1}+n \nu_{2}$ and $\gamma_{21}, \gamma_{11}$ are functions of $\nu_{1}, \nu_{2}$ but not $z$ which can be found in [31]. It follows from (A.9) that

$$
\frac{G_{b}(-z)}{G_{b}(z)}=-e^{+2 z \gamma_{22}} \prod_{m=0}^{\infty} \prod_{n=0}^{\infty}\left(\frac{z+\Omega}{-z+\Omega}\right) e^{-\frac{2 z}{\Omega}} .
$$


In the limit $x \rightarrow \infty$ with $\pm \operatorname{Im}(x)>0$ one finds [31,32],

$$
\ln \left(S_{b}(x)\right)= \pm i \pi\left(\frac{x^{2}}{2}-\frac{Q x}{2}-\frac{1}{2}\left(b^{2}+\frac{1}{b^{2}}+2\right)\right)+o\left(\frac{1}{x}\right)
$$




\section{References}

[1] A. Strominger, "Open string creation by s-branes," arXiv:hep-th/0209090.

[2] F. Larsen, A. Naqvi and S. Terashima, "Rolling tachyons and decaying branes," arXiv:hep-th/0212248.

[3] M. R. Gaberdiel, A. Recknagel and G. M. Watts, "The conformal boundary states for SU(2) at level 1," Nucl. Phys. B 626, 344 (2002) arXiv:hep-th/0108102.

[4] I. Runkel and G. M. Watts, "A non-rational CFT with $\mathrm{c}=1$ as a limit of minimal models," JHEP 0109, 006 (2001) arXiv:hep-th/0107118.

[5] C. G. Callan, I. R. Klebanov, A. W. Ludwig and J. M. Maldacena, "Exact solution of a boundary conformal field theory," Nucl. Phys. B 422, 417 (1994) arXiv:hepth/9402113].

[6] J. Polchinski and L. Thorlacius, "Free Fermion Representation Of A Boundary Conformal Field Theory," Phys. Rev. D 50, 622 (1994) arXiv:hep-th/9404008.

[7] V. Fateev, S. Lukyanov, A. B. Zamolodchikov and A. B. Zamolodchikov, "Expectation values of boundary fields in the boundary sine-Gordon model," Phys. Lett. B 406, 83 (1997) arXiv:hep-th/9702190.

[8] V. Fateev, A. B. Zamolodchikov and A. B. Zamolodchikov, "Boundary Liouville field theory. I: Boundary state and boundary two-point function," arXiv:hep-th/0001012.

[9] J. Teschner, "Remarks on Liouville theory with boundary," arXiv:hep-th/0009138.

[10] K. Hosomichi, "Bulk-boundary propagator in Liouville theory on a disc," JHEP 0111, 044 (2001) arXiv:hep-th/0108093.

[11] B. Ponsot and J. Teschner, "Boundary Liouville field theory: Boundary three point function," Nucl. Phys. B 622, 309 (2002) arXiv:hep-th/0110244.

[12] T. Fukuda and K. Hosomichi, "Super Liouville theory with boundary," Nucl. Phys. B 635, 215 (2002) arXiv:hep-th/0202032.

[13] A. Zamolodchikov, private communication.

[14] A. Sen, "Rolling tachyon," JHEP 0204, 048 (2002) [arXiv:hep-th/0203211.

[15] A. Sen, "Tachyon matter," JHEP 0207, 065 (2002) arXiv:hep-th/0203265.

[16] M. Gutperle and A. Strominger, "Spacelike branes," JHEP 0204, 018 (2002) arXiv:hep-th/0202210.

[17] T. Okuda and S. Sugimoto, "Coupling of rolling tachyon to closed strings," Nucl. Phys. B 647, 101 (2002) arXiv:hep-th/0208196].

[18] J. Maldacena, unpublished note.

[19] S. Minwalla and K. Pappododimas, unpublished.

[20] B. Chen, M. Li and F. L. Lin, "Gravitational radiation of rolling tachyon," JHEP 0211, 050 (2002) arXiv:hep-th/0209222.

[21] A. Maloney, A. Strominger and X. Yin, in progress. 
[22] N. Hatano and D. Nelson, "Vortex Pinning and Non-Hermitian Quantum Mechanics,", Phys. Rev. B 56, 8651 (1997), arXiv:cond-mat/9705290.

[23] I. Affleck and D. Nelson, in progress.

[24] M. Goulian and M. Li, "Correlation Functions In Liouville Theory," Phys. Rev. Lett. 66, 2051 (1991).

[25] Y. Tanii and S. I. Yamaguchi, "Two-dimensional quantum gravity on a disk," Mod. Phys. Lett. A 7, 521 (1992) arXiv:hep-th/9110068.

[26] H. Dorn and H. J. Otto, "Two and three point functions in Liouville theory," Nucl. Phys. B 429, 375 (1994) arXiv:hep-th/9403141.

[27] A. B. Zamolodchikov and A. B. Zamolodchikov, "Structure constants and conformal bootstrap in Liouville field theory," Nucl. Phys. B 477, 577 (1996) arXiv:hepth/9506136].

[28] M. B. Green and M. Gutperle, "Symmetry Breaking at enhanced Symmetry Points," Nucl. Phys. B 460, 77 (1996) arXiv:hep-th/9509171.

[29] A. Recknagel and V. Schomerus, "Boundary deformation theory and moduli spaces of D-branes," Nucl. Phys. B 545, 233 (1999) arXiv:hep-th/9811237.

[30] M. R. Gaberdiel and A. Recknagel, "Conformal boundary states for free bosons and fermions," JHEP 0111, 016 (2001) |arXiv:hep-th/0108238.

[31] E.W Barnes, "Theory of the Double Gamma function", Phil. Trans. Roy.Soc, A196 (1901) 265.

[32] M. Jimbo and T. Miwa, "QKZ equation with $|\mathrm{q}|=1$ and correlation functions of the XXZ model in the gapless regime," J. Phys. A 29, 2923 (1996) arXiv:hep-th/9601135. 